\title{
Presencia de inestabilidad microsatélite en pacientes colombianos con adenocarcinoma colorrectal
}

\section{Microsatellite instability in Colombian patients with colorectal adenocarcinoma}

Omar Gómez-Rodríguez, ${ }^{1}$ Rafael Baracaldo-Ayala, ${ }^{1}$ José Fernando Polo, ${ }^{1}$ Elizabeth Velásquez, ${ }^{2}$ Patricia López-Correa, $, 1,3$

Rafael Parra-Medina. ${ }^{1,3,4^{*}}$ (ib

\begin{abstract}
Gacceso abierto
Citación:

Gómez-Rodríguez 0, Baracaldo-Ayala

$R$, Polo JF, Velásquez E, López-Correa P,

Parra-Medina R. Presencia de inestabilidad microsatélite en pacientes colombianos con adenocarcinoma colorrectal. Rev Colomb Gastroenterol. 2021;36(3):349-357. https://do org/10.22516/25007440.686
\end{abstract}

Departamento de Patología, Fundación Universitaria de Ciencias de la Salud, Hospital de San José de Bogotá, Hospital Infantil de San José de Bogotá. Bogotá, Colombia. Departamento de Cirugía, Fundación Universitaria de Ciencias de la Salud, Hospital de San José de Bogotá, Hospital Infantil de San José de Bogotá. Bogotá, Colombia. Departamento de Patología. Instituto Nacional de Cancerología. Bogotá, Colombia.

División de investigaciones, Fundación Universitaria de Ciencias de la Salud, Hospital de San José de Bogotá, Hospital Infantil de San José de Bogotá. Bogotá, Colombia.

*Correspondencia: Rafael Parra-Medina. rafa.parram@gmail.com

Fecha recibido: $23 / 10 / 20$ Fecha aceptado: 01/03/21

\section{Resumen}

Introducción y objetivos: la vía de inestabilidad de microsatélites (IMS) está implicada en la carcinogénesis de un $15 \%$ de carcinomas colorrectales (CCR). La detección de esta alteración tiene relevancia en el pronóstico y en el tratamiento de los pacientes con CCR. El objetivo del presente estudio es determinar la prevalencia de IMS en CCR en una cohorte de pacientes de Bogotá, Colombia. Materiales y métodos: se evaluó por inmunohistoquímica la presencia de homólogo MutL 1 (MLH1), segregación posmeiótica aumentada 2 (PMS2), homólogo mutS 2 (MSH2) y homólogo mutS 6 (MSH6) en muestras de CCR prevenientes de colectomías. Adicionalmente, se analizaron las variables clinicopatológicas. Alos casos con pérdida de MLH1 y PMS2 se les evaluó la mutación del gen BRAF. Resultados: en total se incluyeron 86 casos. La mediana de edad de los pacientes con CCR fue de 69 años, 52,3\% fueron hombres. De los pacientes con CCR, 12 $(13,9 \%)$ presentaron IMS, de los cuales $10(83,3 \%)$ tenían ausencia de expresión MLH1/PMS2 y 2 (16,7 \%), de MSH2/MSH6. La mediana de edad de los pacientes con CCR e IMS fue de 52 años (45-76,5), 9 eran hombres y el $66,7 \%$ de estos casos se localizaron en el colon derecho. El tipo histológico más frecuente fue adenocarcinoma moderadamente diferenciado (67\%). Los linfocitos infiltrantes al tumor fueron observados en el $83 \%$, la presencia de infiltrado de tipo Crohn estaba presente en el $42 \%$. La mutación de BRAF se observó en el $30 \%$ de los pacientes con pérdida de MLH1 y PMS2. Conclusión: la prevalencia de la IMS en nuestra población fue de $14 \%$, datos similares a los observados en la población norteamericana y europea. Sin embargo, se observa que el $83 \%$ presentó pérdida de expresión del complejo MLH1/PMS2, una prevalencia mayor comparada con otras poblaciones.

\section{Palabras clave}

Colon, inestabilidad microsatélite, Colombia.

\section{Abstract}

Introduction: The microsatellite instability (MSI) pathway is involved in the carcinogenesis of $15 \%$ of colorectal carcinomas $(\mathrm{CRC})$. The detection of this alteration is relevant for the prognosis and treatment of CRC patients. Objective: The aim of this study is to determine the prevalence of MSI in CRC in a cohort of patients in Bogotá, Colombia. Materials and methods: The presence of MLH1, PMS2, MSH2, and MSH6 was evaluated by immunohistochemistry in $\mathrm{CRC}$ samples collected during colectomy. Clinicopathological variables were analyzed as well. Cases with loss of MLH1 and PMS2 were evaluated for BRAF gene mutation. Results: A total of 86 cases were included. The median age was 69 years, $52.3 \%$ were male. $12(13.9 \%)$ patients had IMS, $10(83.3 \%)$ had absence of MLH1/PMS2 expression and 2 (16.7\%) absence of MSH2/MSH6 expression. The median age of patients with IMS was 52 years (45-76.5), of which 9 were male. $66.7 \%$ of carcinomas were located in the right colon and the most frequent histological type was moderately differentiated adenocarcinoma $(67 \%)$. Tumor infiltrating lymphocytes were observed in $83 \%$ of the cases, while the presence of Crohn's-like infiltrate was present in $42 \%$. BRAF mutation was observed in $30 \%$ of patients with loss of MLH1 and PMS2. Conclusion: The prevalence of IMS in our population was $14 \%$, similar to the data observed in the North American and European populations. However, we observed that $83 \%$ had loss of expression of the MLH1/PMS2 complex, a higher prevalence compared to other populations.

Keywords

Colon; Microsatellite instability; Colombia. 


\section{INTRODUCCIÓN}

El carcinoma colorrectal (CCR) ocupa el cuarto lugar a nivel mundial por incidencia de cáncer $(6,1 \%)$ y el segundo lugar $(9,2 \%)$ por muertes por cáncer en 2018 en ambos sexos. La incidencia de CCR en Latinoamérica es baja $(7,5 \% \text { del total })^{(1)}$. En Colombia, el CCR es la tercera causa de mortalidad por cáncer, seguida de los carcinomas de próstata y pulmón ${ }^{(2)}$.

El desarrollo del CCR ocurre con mayor frecuencia de forma esporádica, entre el $70 \%$ y el $80 \%$, seguido de la forma hereditaria, entre el $15 \%$ y el $30 \%{ }^{(3)}$. Menos del $10 \%$ corresponde a la variante heredada, que se divide en dos grupos: la forma no polipósica y la polipósica. El CCR no relacionado con la poliposis (HNPCC) está asociado con mecanismos de reparación del ácido desoxirribonucleico $(\mathrm{ADN})$, como la inestabilidad de microsatélites (IMS), que es la principal causa del síndrome de Lynch (SL) (3\%-4\%). La otra variante (menos de $1 \%$ ) se refiere a la poliposis adenomatosa familiar (PAF), que se caracteriza por la formación de múltiples pólipos potencialmente malignos. Un pequeño subconjunto del $1 \%$ al $2 \%$ de los casos de CCR surge como consecuencia de inflamación crónica (enfermedades inflamatorias intestinales) $)^{(4)}$. Es importante resaltar que los casos de CCR esporádicos no tienen factores de riesgo genéticos identificados; el desarrollo de la enfermedad se debe a factores dietéticos, estilo de vida, factores medioambientales o mutaciones somáticas adquiridas ${ }^{(5)}$; mientras que los casos hereditarios y familiares tienen un inicio clínico más temprano. Las mutaciones se adquieren a lo largo de la vida en los casos familiares y esporádicos, a diferencia de los casos hereditarios, cuya mutación germinal es adquirida en el nacimiento ${ }^{(6)}$. La identificación de tumores esporádicos y hereditarios tiene relevancia para el seguimiento y pronóstico de los pacientes. Es conocido que los tumores hereditarios tienen mayor riesgo de desarrollar otros tumores; por tanto, el seguimiento debe ser más estricto ${ }^{(7)}$.

La IMS es causada por un defecto en las proteínas encargadas de la reparación del mal apareamiento (reparación por mistmach [MMR]: homólogo MutL 1 [MLH1], segregación posmeiótica aumentada 2 [PMS2], homólogo mutS $2[\mathrm{MSH} 2]$ y homólogo mutS $6[\mathrm{MSH} 6]$ homólogo MutL $3[\mathrm{MLH} 3]$, homólogo mutS $3[\mathrm{MSH} 3]$, segregación posmeiótica aumentada $1[P M S 1]$ y exonucleasa $1[$ Exo1]). Estas proteínas forman heterodímeros para corregir alteraciones en el apareamiento de nucleótidos generadas por el clivaje del $\mathrm{ADN}$ anómalo ayudando en la escisión de errores de mal apareamiento y en la formación de nuevas cadenas de ADN corregidas. Las alteraciones de los heterodímeros MLH1/PMS2 y MSH2/MSH6 están asociadas con diferentes tumores como en colon, endometrio, estómago, ovario, próstata, entre otros ${ }^{(8)}$. A nivel clínico, es importante identificar a los pacientes con IMS debido a que tienen mejor pronóstico y una tasa de recurrencia reducidas cuando el tratamiento se ha vinculado con el uso de inmunoterapia, la cual ha demostrado ser superior al manejo con quimioterapia convencional ${ }^{(9,10)}$.

La IMS puede identificarse por inmunohistoquímica (IHQ) o por reacción en cadena de la polimerasa (PCR). Con base en su resultado se puede clasificar en 4 categorías:

1. Defectos de MMR (dMMR) esporádica: hipermetilación de la región promotora de MLH1.

2. Síndrome de Lynch debido a la mutación germinal en uno de los MMR (MLH1, PMS2, MSH2, MSH6) o alteraciones en el gen EpCAM (molécula de adhesión celular epitelial); transductor de señal de calcio asociado a tumor 1 [TACSTD1]) que causa silenciamiento epigenético de $\mathrm{MSH} 2$.

3. Cáncer de colon con dMMR inexplicable (casos sin mutación de línea germinal identificada de MMR ni hipermetilación de la región promotora de MLH1): estos casos tienen dMMR inexplicable y han sido calificados como tipo Lynch.

4. Síndrome de deficiencia MMR constitucional (mutaciones bialélicas de MMR en la línea germinal): en estos casos, el tejido adyacente normal también tiene $\mathrm{dMMR}^{(8)}$.

En Latinoamérica se ha evaluado la prevalencia de IMS en diferentes poblaciones ${ }^{(1-15)}$. En Colombia se cuenta con pocos datos acerca de esta condición en pacientes con CCR; por tal motivo, los objetivos del presente estudio son conocer la prevalencia de IMS en CCR atendidos en dos hospitales de alta complejidad de Colombia y, adicionalmente, describir las características clínico-patológicas. Generalmente, los tumores con IMS son en el colon derecho de grados histológicos altos o con diferenciación mucinosa, y asociado con abundantes linfocitos infiltrantes de tumores (LIT) y una respuesta inmunitaria del huésped similar a la de Crohn (Crohn-like) ${ }^{(5,16,17)}$.

\section{MATERIALES Y MÉTODOS}

\section{Población de estudio}

Se realizó un estudio de corte transversal en el que se determinó la presencia de IMS en CCR a través de IHQ evaluando la pérdida de expresión de las proteínas de MMR (MLH1, PMS2, MSH2 y MSH6) Se revisaron las bases de datos y se realizó un filtro de todos los pacientes diagnosticados y tratados con CCR por colectomía en el Hospital de San José y el Hospital Infantil Universitario de San José 
en Bogotá, Colombia, durante enero de 2012 y diciembre de 2017. No se incluyeron biopsias de CCR teniendo en cuenta que varios pacientes son diagnósticos en estos hospitales; sin embargo, el seguimiento y tratamiento clínico se realizan en otros hospitales; por tanto, no se puede conocer con certeza el estadio tumoral. El criterio de exclusión fue aquellos pacientes para los cuales el material de patología no fue apto para el procesamiento por IHQ. Estos dos hospitales son de alta complejidad y se encuentran ubicados en dos zonas de la capital colombiana, lo que permite tener poblaciones heterogéneas de diferentes etnias y estratos socioeconómicos.

Se evaluaron las siguientes variables clínicas e histológicas: edad, tamaño tumoral, localización tumoral, grado histológico (bien diferenciado [G1], moderadamente diferenciado [G2] y pobremente diferenciado [G3]). Cuando la mucosecreción superó el $50 \%$ de la superficie tumoral se lo consideró como un tumor mucinoso. La presencia de linfocitos intratumorales (LIT) fue medida en 5 campos de gran aumento $(40 \mathrm{x})$ en el frente de invasión tumoral. Se clasificó en 0 (ausencia de linfocitos), 1 (entre 2 y 5 linfocitos) y 3 (mayor de 5 linfocitos); la inflamación tipo Crohn fue definida como la acumulación de linfocitos con o sin formación de centro germinal en el frente de infiltración tumoral, positividad y cantidad de ganglios comprometidos. El tipo histológico y la clasificación TNM (tumor, ganglios y metástasis) se determinó según los criterios de la Organización Mundial de la Salud (OMS) ${ }^{(18)}$. Se revisaron las historias clínicas completas de los pacientes con pérdida de la expresión de las proteínas de MMR en busca de conocer si cumplían con los criterios de síndrome de Lynch con base en la guía Bethesda ${ }^{(19)}$.

\section{Estudio de IHQ}

A todos los casos seleccionados para el estudio se les realizaron cortes a $3 \mu \mathrm{M}$ para el marcado inmunológico con los siguientes anticuerpos primarios: MLH1 (clon: BS29, LabVision Autostainer, fuente e isotipo de la inmunoglobulina [Ig]: inmunoglobulina G1 [IgG1] de ratón, dilución 1:100, presentación: MAD-000726Q); MSH2 (clon: FE11, LabVision Autostainer, fuente e isotipo de la Ig: IgG1 $/ \mathrm{kg}$, dilución 1:50, presentación: MAD-000677Q); PMS2 (clon: EP51, LabVision Autostainer, fuente e isotipo de la Ig: IgG de conejo, dilución 1:50, presentación: MAD000681Q) y MSH6 (clon: EP49, LabVision Autostainer, fuente e isotipo de la Ig: IgG de conejo, dilución 1:50, presentación: MAD-000635Q).

Las secciones del tumor se mantuvieron a $60^{\circ} \mathrm{C}$ durante 2 horas, se desparafinizó en xileno durante 10 minutos y se rehidrató en etanol. La recuperación de antígenos mediada por calor se llevó a cabo utilizando una solución 1/10 EDTA 10X (LabVision ${ }^{\mathrm{rx}}$ ) en un "vaporizador" durante 50 minutos. A continuación, los bloques de parafina se sumergieron en una solución de peróxido de hidrógeno $1 / 10$ (Hydrogen Peroxide Block UltraVision) durante 10 minutos a temperatura ambiente y se incubaron con una solución de anticuerpo primario por 2 horas a temperatura ambiente en una cámara húmeda. Después de dos lavados en TBS 1/10 (solución salina tamponada con Dako Tris, pH: 7,6), los tejidos se incubaron con anticuerpo secundario biotinilado (amplificador de anticuerpos primarios) durante 10 minutos a temperatura ambiente. El desarrollo del color se realizó con tampón DBA y los portaobjetos se mantuvieron con hematoxilina durante dos minutos. Se usó una sección en la que se realizó un procedimiento inmunohistoquímico sin agregar el anticuerpo primario como control negativo. Los portaobjetos de control positivo incluyeron muestras de CCR.

El estudio de IHQ se efectuó y su resultado fue revisado por dos patólogos (PL, FP) mediante microscopia de luz, categorizando reactividad o no reactividad de cada uno de los marcadores, en caso de encontrar pérdida de expresión (no reactividad) de los marcadores MLH1, MSH2, MSH6 y PMS2. El control positivo fue la tinción nuclear en la mucosa normal o la infiltración linfocítica.

\section{Análisis de mutación de BRAF V600}

Con base en los algoritmos para determinar CCR somáticos y hereditarios ${ }^{(20)}$, se le realizó PCR y secuenciación para detectar mutación V600 (p.Val600Glu, p.Val600Asp, p.Val600Lys y p.Val600Arg) en el gen BRAF (NM_004333.4, chr. 7) a los casos con pérdida de MLH1 evaluado por IHQ.

\section{Análisis estadístico}

El análisis estadístico se realizó resumiendo las variables cuantitativas con medianas y rangos intercuartílicos (RIC). Las asociaciones entre la presencia de IMS y las características clínico-patológicas se evaluaron mediante la prueba exacta de Fisher. Se asumió significancia estadística con valores $p<0,05$.

\section{Consideraciones éticas}

El estudio fue aprobado por los comités de ética de investigación humana de la Fundación Universitaria de Ciencias de la Salud y fue realizado de acuerdo con los principios de la Declaración de Helsinki. 


\section{RESULTADOS}

\section{Población con CCR}

En total se incluyeron 86 pacientes, de los cuales el 52,3\% fueron hombres. La mediana de edad al momento del procedimiento quirúrgico fue de 69 años (RIC: 59-77). 39 casos $(45,35 \%)$ presentaron el tumor en el colon derecho, $33(38,4 \%)$ entre el sigmoide y el recto, $8(9,3 \%)$ en el colon izquierdo, $7(8,14 \%)$ en el transverso y un caso $(1,2 \%)$ presentó tumores sincrónicos de localización en el colon derecho y transverso (Tabla $\mathbf{1}$ ).

Tabla 1. Características clínico-patológicas de pacientes con CCR

\begin{tabular}{|c|c|c|c|c|}
\hline Características & $\begin{array}{c}\text { Total }(\%) \\
n=86\end{array}$ & $\begin{array}{c}\text { EMS }(\%) \\
n=74\end{array}$ & $\begin{array}{c}\operatorname{IMS}(\%) \\
n=12\end{array}$ & Valor $p$ \\
\hline Edad, mediana (RIC) & $69(59-77)$ & $69(60-78)$ & $52(45-76,5)$ & 0,0606 \\
\hline $\begin{array}{l}\text { Sexo } \\
\text { - Masculino } \\
\text { - Femenino }\end{array}$ & $\begin{array}{l}45(52,3) \\
41(47,7)\end{array}$ & $\begin{array}{l}36(48,6) \\
38(51,4)\end{array}$ & $\begin{array}{l}9(75) \\
3(25)\end{array}$ & 0,090 \\
\hline Tamaño tumoral (RIC) & $5(3-7)$ & $4,25(3-7)$ & $6(4,75-8,5)$ & 0,1330 \\
\hline $\begin{array}{l}\text { Localización } \\
\text { - Colon ascendente (derecho y transverso) } \\
\text { - Colon descendente (izquierdo y sigmoide) y recto }\end{array}$ & $\begin{array}{l}46(52,8) \\
41(47,2)\end{array}$ & $\begin{array}{c}37(42,5) \\
37(49)\end{array}$ & $\begin{array}{c}8(10) \\
2(3)\end{array}$ & 0,180 \\
\hline $\begin{array}{l}\text { Tipo histológico } \\
\text { - Adenocarcinoma convencional } \\
\text { - Mucinoso } \\
\text { - Anillo de sello } \\
\text { - Adenoescamoso }\end{array}$ & $\begin{array}{l}71(82,6) \\
12(13) \\
2(2,3) \\
1(1,2)\end{array}$ & $\begin{array}{c}62(83,8) \\
10(12,2) \\
2(2,7) \\
1(1,3)\end{array}$ & $\begin{array}{c}8(72,7) \\
3(25) \\
0 \\
1(8,3)\end{array}$ & 0,516 \\
\hline $\begin{array}{l}\text { Grado histológico de los adenocarcinomas convencionales } \\
\text { - Bien diferenciado } \\
\text { - Moderado } \\
\text { - Mal diferenciado }\end{array}$ & $\begin{array}{c}5(7) \\
59(83,1) \\
7(9,8)\end{array}$ & $\begin{array}{c}5(7) \\
51(71,8) \\
6(8,4)\end{array}$ & $\begin{array}{c}0 \\
8(11,2) \\
1(1,4)\end{array}$ & 0,735 \\
\hline Multifocalidad & $9(10,5)$ & $8(10,8)$ & $1(8,3)$ & 0,795 \\
\hline $\begin{array}{l}\text { pT } \\
\text { - Lámina propia/muscular de la mucosa } \\
\text { - Submucosa } \\
\text { - Muscular propia } \\
\text { - Hasta la subserosa } \\
\text { - Serosa } \\
\text { - Otros órganos }\end{array}$ & $\begin{array}{c}3(3,4) \\
6(6,9) \\
19(22,1) \\
2(2,3) \\
53(61,6) \\
3(3,5)\end{array}$ & $\begin{array}{c}3(4) \\
5(6,8) \\
13(17,6) \\
2(2,7) \\
49(66,2) \\
2(2,7)\end{array}$ & $\begin{array}{c}0 \\
1(8,3) \\
6(50) \\
0 \\
4(33,3) \\
1(8,3)\end{array}$ & 0,130 \\
\hline $\begin{array}{l}\mathrm{pN} \\
-\mathrm{N} 0 \\
-\mathrm{N} 1 \mathrm{a} \\
-\mathrm{N} 1 \mathrm{~b} \\
-\mathrm{N} 2 \mathrm{a} \\
-\mathrm{N} 2 \mathrm{~b}\end{array}$ & $\begin{array}{c}28(32,6) \\
58(67,4) \\
8(9,3) \\
10(11,6) \\
4(4,6) \\
0\end{array}$ & $\begin{array}{c}27(36,5) \\
47(63,5) \\
8(10,8) \\
9(12,1) \\
4(5,4) \\
0\end{array}$ & $\begin{array}{c}1(8,3) \\
11(91,7) \\
0 \\
1(8,3) \\
0 \\
0\end{array}$ & 0,054 \\
\hline Depósitos tumorales & $3(3,5)$ & $3(3,5)$ & 0 & 0,478 \\
\hline Infiltrado de tipo Crohn & $18(20,9)$ & $13(17,6)$ & $5(41,7)$ & 0,057 \\
\hline $\begin{array}{l}\text { Linfocitos infiltrantes del tumor } \\
\text { - Ausente } \\
\text { - Leve-moderado } \\
\text { - Marcado }\end{array}$ & $\begin{array}{l}22(25,6) \\
54(62,8) \\
10(11,6)\end{array}$ & $\begin{array}{c}20(27,0) \\
46(62,2) \\
8(10,8)\end{array}$ & $\begin{array}{l}2(16,7) \\
8(66,7) \\
2(16,7)\end{array}$ & 0,680 \\
\hline
\end{tabular}

EMS: estabilidad microsatélite. 
Los subtipos histológicos observados fueron 71 (82,5\%) pacientes con adenocarcinomas de tipo convencional, 12 $(13,9 \%)$ presentaron adenocarcinoma mucinoso, 2 mostraron la variante en anillo de sello $(2,3 \%)$ y 1 se asoció con el subtipo adenoescamoso y mucinoso (1,2 \%), previamente publicado ${ }^{(21)}$. En cuanto al grado de diferenciación de los adenocarcinomas convencionales, 59 (83,1\%) fueron clasificados como tumores moderadamente diferenciados, 7 (9,8\%) pobremente diferenciados y $5(7 \%)$ bien diferenciados. Es importante resaltar que el 32,6\% presentó metástasis a ganglios linfáticos. En el 3,49\% se encontraron depósitos tumorales (Tabla 1).

\section{Población con CCR e IMS}

En total, $12(13,9 \%)$ pacientes presentaron IMS, todos con IMS alta (definida por la ausencia de expresión de más de dos proteínas MMR) (Tabla 2). 10 (83,3 \%) pacientes presentaron ausencia de MLH1 y PMS2, y 2 (16,7 \%) de MSH2 y MSH6. Se presentaron 9 casos en hombres y 3 en mujeres, y la mediana de edad fue de 52 años (RIC: 45-76,5). La mutación de BRAF fue observada en el $30 \%$ de los pacientes con pérdida de MLH1 y PMS2.

La localización más frecuente fue en el colon derecho (8/12: 66,7\%), seguido de sigmoide y recto (2/12: 16,7\%). El tipo histológico más frecuente fue adenocarcinoma moderadamente diferenciado $(8 / 12)$, seguido de adenocarcinoma mucinoso $(3 / 12)$ y un caso adenoescamoso y mucinoso. Se reconocieron $10 / 12$ casos $(83,3 \%)$ de LIT acompañantes, 8 de leves a moderados y 2 marcados. Además, 5/12 casos (41,7\%) presentaron infiltrado de tipo Crohn. 6/12 (50\%) pacientes tenían infiltración hasta la muscular propia ( $\mathrm{pT} 2 \mathrm{NM})$, seguido de infiltración hasta la serosa 4/12 (33,3\%) (pT4aNM), 1/12 (8,3\%) hasta la

Tabla 2. Características clínicas y patológicas de los pacientes con IMS

\begin{tabular}{|c|c|c|c|c|c|c|c|c|c|c|c|c|c|}
\hline \multirow{2}{*}{$\begin{array}{l}\mathrm{N}^{0} \text { de } \\
\text { casos }\end{array}$} & \multirow{2}{*}{ Edad } & \multirow[t]{2}{*}{ Sexo } & \multirow{2}{*}{$\begin{array}{c}\text { Colon } \\
\text { derecho }\end{array}$} & \multirow{2}{*}{$\begin{array}{l}\text { Diferen- } \\
\text { ciación } \\
\text { mucinosa }\end{array}$} & \multirow{2}{*}{$\begin{array}{l}\text { Linfocitos } \\
\text { infiltrantes } \\
\text { del tumor }\end{array}$} & \multirow{2}{*}{$\begin{array}{c}\text { Reacción } \\
\text { linfocítica } \\
\text { tipo } \\
\text { Crohn }\end{array}$} & \multicolumn{4}{|c|}{ Expresión anormal } & \multirow{2}{*}{$\begin{array}{l}\text { Mutación } \\
\text { del gen } \\
\text { BRAF V600 }\end{array}$} & \multirow{2}{*}{$\begin{array}{l}\text { ¿Cumple con } \\
\text { los criterios } \\
\text { de síndrome } \\
\text { de Lynch? }\end{array}$} & \multirow{2}{*}{$\begin{array}{l}\text { Estadio } \\
\text { clínico }\end{array}$} \\
\hline & & & & & & & MLH1 & MSH2 & MSH6 & PMS2 & & & \\
\hline 1 & 21 & Hombre & No & Sí & Sí & No & Sí & No & No & Sí & No & No & $\begin{array}{l}\text { T3NOMO } \\
\text { estadio Ila }\end{array}$ \\
\hline 2 & 36 & Mujer & No & No & Sí & No & Sí & No & No & Sí & No & No & $\begin{array}{l}\text { T3NOMO } \\
\text { estadio Ila }\end{array}$ \\
\hline 3 & 43 & Hombre & Sí & Sí & No & No & Sí & No & No & Sí & Sí & No & $\begin{array}{l}\text { T2N0MO } \\
\text { estadio I }\end{array}$ \\
\hline 4 & 48 & Hombre & Sí & No & Sí & Sí & No & Sí & Sí & No & No & Sí & $\begin{array}{l}\text { T3NOMO } \\
\text { estadio lla }\end{array}$ \\
\hline 5 & 50 & Hombre & Sí & No & Sí & No & Sí & No & No & Sí & No & No & $\begin{array}{l}\text { T4aNOM0 } \\
\text { estadio Ilb }\end{array}$ \\
\hline 6 & 51 & Hombre & Sí & No & Sí & Sí & Sí & No & No & Sí & No & No & $\begin{array}{l}\text { T4BNOM0 } \\
\text { estadio IIC }\end{array}$ \\
\hline 7 & 53 & Hombre & No & No & Sí & No & Sí & No & No & Sí & No & No & $\begin{array}{l}\text { T2NOMO } \\
\text { estadio I }\end{array}$ \\
\hline 8 & 61 & Mujer & Sí & No & Sí & No & Sí & No & No & Sí & Sí & No & $\begin{array}{l}\text { T3N0MO } \\
\text { estadio lla }\end{array}$ \\
\hline 9 & 76 & Hombre & Sí & Sí & Sí & Sí & No & Sí & Sí & No & No & Sí & $\begin{array}{l}\text { T2NOMO } \\
\text { estadio I }\end{array}$ \\
\hline 10 & 77 & Mujer & Sí & No & Sí & Sí & Sí & No & No & Sí & Sí & No & $\begin{array}{l}\text { T2N0M0 } \\
\text { estadio I }\end{array}$ \\
\hline 11 & 82 & Hombre & Sí & No & Sí & Sí & Sí & No & No & Sí & Sí & No & $\begin{array}{l}\text { T2N0M0 } \\
\text { estadio I }\end{array}$ \\
\hline 12 & 82 & Hombre & No & No & Sí & No & Sí & No & No & Sí & No & No & $\begin{array}{l}\text { T3N0M0 } \\
\text { estadio Ila }\end{array}$ \\
\hline
\end{tabular}


lámina propia (pTisNM) y $1 / 12(8,3 \%)$ con infiltración hasta otros órganos (estómago). Solo $1 / 12$ presentó compromiso a ganglios linfáticos (pTN1bM) (Tablas 1 y 2). 5 pacientes fueron categorizados en estadio IIA, 5 pacientes en estadio I, 1 paciente en estadio IIB y 1 paciente en estadio IIC, con base en la clasificación del Comité Conjunto Americano del Cáncer (AJCC), en su octava edición. Según la guía de Bethesda ${ }^{(19)}$, dos pacientes cumplían con los criterios de síndrome de Lynch.

\section{DISCUSIÓN}

La prevalencia de IMS en pacientes con CCR puede variar entre las poblaciones, lo cual podría obedecer a características medioambientales o factores genéticos. Los datos reportados en diferentes estudios multicéntricos informan que aproximadamente del $10 \%$ al $15 \%$ de los pacientes se encuentran en estadios II-III y el $5 \%$ en estadio IV ${ }^{(22-26)}$; nosotros encontramos una prevalencia de IMS alta en el $14 \%$ de los CCR.

En Latinoamérica se ha reportado la prevalencia de IMS en CCR en diferentes poblaciones en México (21,3\%), Brasil (23 \%), Perú $(38,4 \%)$ y Argentina $(45 \%)^{(11-15)}$. Además, otro estudio desarrollado en población latina de Estados Unidos encontró una prevalencia del 12,6\% $(\mathrm{n}=111)^{(27)}$.

En Colombia se han realizado pocos estudios. Por una parte, el único estudio similar al presente en el que se evaluó a través de IHQ la presencia de IMS fue el publicado por Shamekh y colaboradores ${ }^{(28)}$, quienes encontraron en 45 pacientes con CCR del suroccidente de Colombia la presencia de IMS en 11 pacientes ( $24 \%), 5$ de ellos con pérdida de MLH1/PMS2, 4 con pérdida aislada de PMS2, 1 con pérdida aislada de MLH1 y 1 caso con pérdida de MSH6 y PMS2. Por otra parte, Montenegro y colaboradores ${ }^{(29)}$ realizaron un estudio multicéntrico en el que evaluaron en la sangre periférica la presencia de 6 marcadores microsatelitales (BAT-25, BAT-26, BAT-40, D17S250, D2S123 y D5S346). Buscaron la presencia de estos marcadores en 10 pacientes con CCR esporádico y en 31 pacientes con cáncer de colon hereditario no polipósico. El 34,1 \% presentó IMS, y el $76 \%$, IMS alta. Además, encontraron un nuevo polimorfismo, C399T, en el exón 3 del gen MSH2. Por otra parte, Cárdenas y colaboradores ${ }^{(30)}$ en el nororiente determinaron la presencia de IMS evaluando el marcador BAT-26 en 11 pacientes con CCR, y se reportó dicha presencia en 3 pacientes (27\%). Afanador y colaboradores ${ }^{(31)}$ evaluaron la presencia de 5 marcadores (BAT-25, BAT-26, NR21, NR24 y NR27) por PCR en 39 pacientes con CCR esporádico. En total, se encontró una prevalencia de IMS del 35,9\% (14/39): 12,8 \% (5/39) con IMS alta y 23,1 \% (9/39) con IMS baja.

Las alteraciones en las proteínas de los genes MMR valoradas de manera individual indican que las proteínas
MSH2 y MLH1 representan la mayor parte de las alteraciones en los CCR con IMS a nivel global, con una frecuencia de $40-60 \%$ y $40-50 \%$, respectivamente, mientras que se han encontrado cifras menores para MSH6 (10 - 20\%) y PMS2 $(2 \%)^{(32)}$. Con respecto a estas alteraciones en el presente estudio se encontró un proporción mayor de casos con pérdida de expresión de los complejos MLH1/PMS2 comparado con MSH2/MSH6 ( $83 \%$ y 17\%, respectivamente), resultados discordantes a los publicados en la población de Asia occidental en donde se encontró una proporción equivalente entre estos dos complejos MLH1/PMS2 versus MSH2/MSH6 (56.3\% y 43.8\%, respectivamente) ${ }^{(25)}$, igualmente con casos reportados en Australia (58.3\% y $41.7 \%$, respectivamente) ${ }^{(15)} \mathrm{y}$ en los individuos latinos en los Estados Unidos (44.4\% y 55.6\%, respectivamente) $)^{(27)}$. En México realizaron un análisis de manera individual de la expresión de las proteínas MMR en el que encontraron ausencia de expresión de MLH1 (72\%), PMS2 (62,7 \%), MSH2 (21\%) y MSH6 (23,2 \%); por lo que se desconoce el análisis por complejos MSH1/MSH2 y MSH2/ MSH $^{(11)}$. Sin embargo, nuestro estudio y el publicado previamente por Shamekh y colaboradores ${ }^{(28)}$ sugieren que la alteración de MLH1/PMS2 podría ser la más frecuente en nuestra población. La causa de esta expresión no es clara, pero se asocia con la combinación de factores genéticos, epigenéticos y ambientales ${ }^{(33)}$.

A lo largo de los últimos años, el Colegio Americano de Patología (CAP) ha modificado las indicaciones del estudio de IMS en CCR; de hecho, en algunos centros se les realiza a todos los pacientes. El CAP recomienda realizar el estudio en pacientes menores de 70 años, tumores de localización derecha, tumores con aumento de linfocitos infiltrantes al tumor y con infiltrado de tipo Crohn en subtipos medulares o en anillo de sello, en casos con heterogeneidad intratumoral (carcinoma mixto convencional, mucinoso y pobremente diferenciado), histología de alto grado y ausencia de necrosis sucia ${ }^{(34)}$. Con base en estos resultados, nosotros también recomendamos realizar el estudio de IMS a todos los casos con CCR, teniendo en cuenta que en nuestros pacientes encontramos casos con IMS en colon transverso, descendente y recto; $y$, además, con un grado histológico moderadamente diferenciado.

\section{CONCLUSIÓN}

En conclusión, la prevalencia de IMS asociada con CCR en nuestra población es del $14 \%$, datos similares a los reportados en la población de Norteamérica y Europa; aunque es una prevalencia menor en comparación con otros países latinoamericanos. Es para resaltar que el $83 \%$ presentó pérdida de la expresión del complejo MLH1/PMS2, un dato mucho mayor comparado con los demás países. Estos resul- 
tados deberían ser el inicio para generar estudios con una mayor cantidad de pacientes $y$, de esta manera, conocer la prevalencia de esta condición clínica en Colombia. Esto va a permitir tomar conductas clínicas en nuestra población, considerando que la IMS en CCR tiene repercusión en el pronóstico, tratamiento y seguimiento de los pacientes.

\section{Agradecimientos}

Agradecemos al personal de apoyo y técnicos en histología del Hospital de San José y al Hospital Universitario Infantil de San José, de la Fundación Universitaria de Ciencias de la Salud, quienes ayudaron a recopilar los materiales necesarios para los procesos realizados en este estudio.

\section{Fuentes de financiación}

Este estudio fue posible gracias a los recursos de financiamiento proporcionados por la Escuela de Medicina de la Fundación Universitaria de Ciencias de la Salud.

\section{REFERENCIAS}

1. Ferlay J, Colombet M, Soerjomataram I, Mathers C, Parkin DM, Piñeros M, Znaor A, Bray F. Estimating the global cancer incidence and mortality in 2018: GLOBOCAN sources and methods. Int J Cancer. 2019;144(8):1941-1953. https://doi.org/10.1002/ijc.31937

2. Global Burden of Disease Cancer Collaboration, Fitzmaurice C, Dicker D, Pain A, Hamavid H, MoradiLakeh M, MacIntyre MF, Allen C, Hansen G, Woodbrook R, Wolfe C, Hamadeh RR, Moore A, Werdecker A, Gessner BD, Te Ao B, McMahon B, Karimkhani C, Yu C, Cooke GS, Schwebel DC, Carpenter DO, Pereira DM, Nash D, Kazi DS, De Leo D, Plass D, Ukwaja KN, Thurston GD, Yun Jin K, Simard EP, Mills E, Park EK, Catalá-López F, deVeber G, Gotay C, Khan G, Hosgood HD 3rd, Santos IS, Leasher JL, Singh J, Leigh J, Jonas JB, Sanabria J, Beardsley J, Jacobsen KH, Takahashi K, Franklin RC, Ronfani L, Montico M, Naldi L, Tonelli M, Geleijnse J, Petzold M, Shrime MG, Younis M, Yonemoto N, Breitborde N, Yip P, Pourmalek F, Lotufo PA, Esteghamati A, Hankey GJ, Ali R, Lunevicius R, Malekzadeh R, Dellavalle R, Weintraub R, Lucas R, Hay R, Rojas-Rueda D, Westerman R, Sepanlou SG, Nolte S, Patten S, Weichenthal S, Abera SF, Fereshtehnejad SM, Shiue I, Driscoll T, Vasankari T, Alsharif U, RahimiMovaghar V, Vlassov VV, Marcenes WS, Mekonnen W, Melaku YA, Yano Y, Artaman A, Campos I, MacLachlan J, Mueller U, Kim D, Trillini M, Eshrati B, Williams HC, Shibuya K, Dandona R, Murthy K, Cowie B, Amare AT, Antonio CA, Castañeda-Orjuela C, van Gool CH, Violante F, Oh IH, Deribe K, Soreide K, Knibbs L, Kereselidze M, Green M, Cardenas R, Roy N, Tillmann T, Li Y, Krueger H, Monasta L, Dey S, Sheikhbahaei S, Hafezi-Nejad N, Kumar GA, Sreeramareddy CT, Dandona L, Wang H, Vollset SE, Mokdad A, Salomon JA, Lozano R, Vos T, Forouzanfar M, Lopez A, Murray C, Naghavi M. The Global Burden of Cancer 2013. JAMA Oncol. 2015;1(4):505-27. https://doi.org/10.1001/jamaoncol.2015.0735

3. Ribic CM, Sargent DJ, Moore MJ, Thibodeau SN, French AJ, Goldberg RM, Hamilton SR, Laurent-Puig P, Gryfe R, Shepherd LE, Tu D, Redston M, Gallinger S. Tumor microsatellite-instability status as a predictor of benefit from fluorouracil-based adjuvant chemotherapy for colon cancer. N Engl J Med. 2003;349(3):247-57. https://doi.org/10.1056/NEJMoa022289

4. Xiao H, Yoon YS, Hong SM, Roh SA, Cho DH, Yu CS, Kim JC. Poorly differentiated colorectal cancers: correlation of microsatellite instability with clinicopathologic features and survival. Am J Clin Pathol. 2013;140(3):341-7. https://doi.org/10.1309/AJCP8P2DYNKGRBVI

5. Slattery ML, Curtin K, Anderson K, Ma KN, Ballard L, Edwards S, Schaffer D, Potter J, Leppert M, Samowitz WS. Associations between cigarette smoking, lifestyle factors, and microsatellite instability in colon tumors. J Natl Cancer Inst. 2000;92(22):1831-6. https://doi.org/10.1093/jnci/92.22.1831

6. Liu D. Handbook of Tumor Syndromes. 1. ${ }^{\mathrm{a}}$ edición. CRC Press; 2020. https://doi.org/10.1201/9781351187435

7. Souglakos J. Genetic alterations in sporadic and hereditary colorectal cancer: implementations for screening and follow-up. Dig Dis. 2007;25(1):9-19. https://doi.org/10.1159/000099166

8. Chen W, Swanson BJ, Frankel WL. Molecular genetics of microsatellite-unstable colorectal cancer for pathologists. Diagn Pathol. 2017;12(1):24. https://doi.org/10.1186/s13000-017-0613-8

9. Le DT, Uram JN, Wang H, Bartlett BR, Kemberling H, Eyring AD, Skora AD, Luber BS, Azad NS, Laheru D, Biedrzycki B, Donehower RC, Zaheer A, Fisher GA, Crocenzi TS, Lee JJ, Duffy SM, Goldberg RM, de la Chapelle A, Koshiji M, Bhaijee F, Huebner T, Hruban RH, Wood LD, Cuka N, Pardoll DM, Papadopoulos N, Kinzler KW, Zhou S, Cornish TC, Taube JM, Anders RA, Eshleman JR, Vogelstein B, Diaz LA Jr. PD-1 Blockade in Tumors with Mismatch-Repair Deficiency. N Engl J Med. 2015;372(26):2509-20. https://doi.org/10.1056/NEJMoa1500596

10. Tiwari AK, Roy HK, Lynch HT. Lynch syndrome in the 21st century: clinical perspectives. QJM. 2016;109(3):151-8. https://doi.org/10.1093/qjmed/hcv137 
11. López-Correa PE, Lino-Silva LS, Gamboa-Domínguez A, Zepeda-Najar C, Salcedo-Hernández RA. Frequency of Defective Mismatch Repair System in a Series of Consecutive Cases of Colorectal Cancer in a National Cancer Center. J Gastrointest Cancer. 2018;49(3):379-384. https://doi.org/10.1007/s12029-018-0132-1

12. Leite SM, Gomes KB, Pardini VC, Ferreira AC, Oliveira VC, Cruz GM. Assessment of microsatellite instability in colorectal cancer patients from Brazil. Mol Biol Rep. 2010;37(1):375-80. https://doi.org/10.1007/s11033-009-9807-9

13. Egoavil CM, Montenegro P, Soto JL, Casanova L, SanchezLihon J, Castillejo MI, Martinez-Canto A, Perez-Carbonell L, Castillejo A, Guarinos C, Barbera VM, Jover R, Paya A, Alenda C. Clinically important molecular features of Peruvian colorectal tumours: high prevalence of DNA mismatch repair deficiency and low incidence of KRAS mutations. Pathology. 2011;43(3):228-33. https://doi.org/10.1097/PAT.0b013e3283437613

14. Ortiz C, Dongo-Pflucker K, Martín-Cruz L, Barletta Carrillo C, Mora-Alferez P, Arias A. Inestabilidad de microsatélites en pacientes con diagnóstico de cáncer colorrectal. Rev Gastroenterol Perú. 2016;36(1):15-22.

15. Vaccaro CA, Carrozzo JE, Mocetti E, Berho M, Valdemoros P, Mullen E, Oviedo M, Redal MA. Expresión inmunohistoquímica e inestabilidad microsatelital en el síndrome de Lynch. Medicina (B Aires). 2007;67(3):274-8.

16. Vilar E, Gruber SB. Microsatellite instability in colorectal cancer-the stable evidence. Nat Rev Clin Oncol. 2010;7(3):153-62. https://doi.org/10.1038/nrclinonc.2009.237

17. Baracaldo R, Peña L, Gómez O, Polo JF, López P, ParraMedina R. Características histopatológicas del carcinoma colorrectal con inestabilidad microsatelital (IMS). Repert Med Cir. 2019;29(1):32-40. https://doi.org/10.31260/RepertMedCir.v29.n1.2020.172

18. Bosman FT, Carneiro F, Hruban RH, Theise ND. WHO classification of tumours of the digestive system. World Health Organization; 2010.

19. Giardiello FM, Allen JI, Axilbund JE, Boland CR, Burke CA, Burt RW, Church JM, Dominitz JA, Johnson DA, Kaltenbach T, Levin TR, Lieberman DA, Robertson DJ, Syngal S, Rex DK; US Multi-Society Task Force on Colorectal Cancer. Guidelines on genetic evaluation and management of Lynch syndrome: a consensus statement by the US Multi-Society Task Force on colorectal cancer. Gastroenterology. 2014;147(2):502-26. https://doi.org/10.1053/j.gastro.2014.04.001

20. Paredes SR, Chan C, Rickard MJFX. Immunohistochemistry in screening for heritable colorectal cancer: what to do with an abnormal result. ANZ J Surg. 2020;90(5):702-707. https://doi.org/10.1111/ans.15586

21. Parra-Medina R, Lopez-Correa P, Gutierrez V, Polo F. Colonic adenosquamous carcinoma and mucinous adeno- carcinoma with microsatellite instability. Malays J Pathol. 2018;40(2):199-202.

22. Satia JA, Keku T, Galanko JA, Martin C, Doctolero RT, Tajima A, Sandler RS, Carethers JM. Diet, lifestyle, and genomic instability in the North Carolina Colon Cancer Study. Cancer Epidemiol Biomarkers Prev. 2005;14(2):429-36. https://doi.org/10.1158/1055-9965.EPI-04-0486

23. Hampel H, Frankel WL, Martin E, Arnold M, Khanduja K, Kuebler P, Nakagawa H, Sotamaa K, Prior TW, Westman J, Panescu J, Fix D, Lockman J, Comeras I, de la Chapelle A. Screening for the Lynch syndrome (hereditary nonpolyposis colorectal cancer). N Engl J Med. 2005;352(18):1851-60. https://doi.org/10.1056/NEJMoa043146

24. Buchanan DD, Clendenning M, Rosty C, Eriksen SV, Walsh MD, Walters RJ, Thibodeau SN, Stewart J, Preston S, Win AK, Flander L, Ouakrim DA, Macrae FA, Boussioutas A, Winship IM, Giles GG, Hopper JL, Southey MC, English D, Jenkins MA. Tumor testing to identify lynch syndrome in two Australian colorectal cancer cohorts. J Gastroenterol Hepatol. 2017;32(2):427-438. https://doi.org/10.1111/jgh.13468

25. Alqahtani M, Grieu F, Carrello A, Amanuel B, Mashour M, Alattas R, Alsaleh K, Alsheikh A, Alqahtani S, Iacopetta B. Screening for Lynch Syndrome in Young Colorectal Cancer Patients from Saudi Arabia Using Microsatellite Instability as the Initial Test. Asian Pac J Cancer Prev. 2016;17(4):1917-23. https://doi.org/10.7314/apjcp.2016.17.4.1917

26. Gelsomino F, Barbolini M, Spallanzani A, Pugliese G, Cascinu $\mathrm{S}$. The evolving role of microsatellite instability in colorectal cancer: A review. Cancer Treat Rev. 2016;51:19-26. https://doi.org/10.1016/j.ctrv.2016.10.005

27. Gupta S, Ashfaq R, Kapur P, Afonso BB, Nguyen TP, Ansari F, Boland CR, Goel A, Rockey DC. Microsatellite instability among individuals of Hispanic origin with colorectal cancer. Cancer. 2010;116(21):4965-72. https://doi.org/10.1002/cncr.25486

28. Shamekh R, Cives M, Mejia J, Coppola D. Higher frequency of isolated PMS2 loss in colorectal tumors in Colombian population: preliminary results.2016;8:37-41. https://doi.org/10.2147/PLMI.S94771

29. Montenegro M Y, Ramírez-Castro JL, Isaza J LF, Bedoya B G, Muñetón-Peña CM. Análisis genético en pacientes con cáncer colorrectal. Revista Médica de Chile. 2006; 134(10):1221-9. https://doi.org/10.4067/S0034-98872006001000002

30. Cárdenas W CA, Vargas C, Moreno O, Insuasti J. Análisis de la inestabilidad de microsatélites mediante el marcador BAT26 en una muestra de pacientes del Hospital Universitario de Santander con diagnóstico de cáncer gástrico o colorrectal. Colombia Médica. 2008;39(Supl 2):41-51.

31. Afanador Ayala $\mathrm{CH}$, Palacio Rúa KA, Isaza Jiménez LF, Ahumada Rodríguez E, Ocampo CM, Muñetón Peña CM. Análisis de inestabilidad microsatelital en individuos 
con cáncer colorrectal esporádico del departamento de Antioquia. Revista Colombiana de Cancerología. 2017;21(1):51-2.

https://doi.org/10.1016/j.rccan.2017.02.017

32. Terdiman JP. It is time to get serious about diagnosing Lynch syndrome (hereditary nonpolyposis colorectal cancer with defective DNA mismatch repair) in the general population. Gastroenterology. 2005;129(2):741-4. https://doi.org/10.1016/j.gastro.2005.06.033

33. Ashktorab H, Smoot DT, Carethers JM, Rahmanian M, Kittles R, Vosganian G, Doura M, Nidhiry E, Naab T, Momen B, Shakhani S, Giardiello FM. High incidence of microsatellite instability in colorectal cancer from African Americans. Clin Cancer Res. 2003;9(3):1112-7.

34. Kakar S, Shi C, Berho ME, Driman DK, Fitzgibbons P, Frankel WL, Hill KA, Jessup J, Krasinskas AMN, Washington MK. Protocol for the Examination of Specimens From Patients With Primary Carcinoma of the Colon and Rectum [internet]. College of American Pathologists; 2017 [consultado el 15de junio de 2020]. Disponible en: https://documents.cap.org/protocols/cpgilower-colonrectum-17protocol-4010.pdf 